\title{
USE OF MOBILE FITNESS-RELATED APPLICATIONS AND ACTIVE VIDEO GAMES IN HIGH-SCHOOL YOUTH
}

\author{
KRZYSZTOF SAS-NOWOSIELSKI ${ }^{1}$, SYLWIA SZOPA², AGNIESZKA KOWALCZYK² \\ ${ }^{1}$ Jerzy Kukuczka Academy of Physical Education in Katowice, Faculty of Physical Education, Department of \\ Humanistic Foundations of Physical Culture, Division of Pedagogy and Psychology \\ ${ }^{2}$ Doctoral Studies, Jerzy Kukuczka Academy of Physical Education in Katowice, Faculty of Physical Education \\ Mailing address: Krzysztof Sas-Nowosielski, Jerzy Kukuczka Academy of Physical Education, \\ Faculty of Physical Education, 72a Mikołowska Street, 40-065 Katowice, tel.: +48 32 2075320, fax: +48 22 8651080, \\ e-mail: k.sas-nowosielski@awf.katowice.pl
}

\begin{abstract}
Introduction. Not many studies have been performed in Poland on using mobile applications from the sport and fitness category and exergames. The main purpose of this study was to investigate to what extent such technologies are used by youth. Material and methods. A total of 435 pupils (including 263 females) aged $17.78 \pm 1.19$ years took part in the study. A diagnostic survey was carried out; the data were collected using a questionnaire developed by the authors and a scale assessing the stage of exercise change developed by Marcus, Rakowski, and Rossi (1992). Descriptive statistics (means and standard deviations) were used to describe the data and to test differences between groups; $\mathrm{chi}^{2}$ test was used with Cramér's $\mathrm{V}$ statistic as a measure of effect size in contingency tables larger than 2 x 2. Results. Of the 435 participants, approximately a third (30.8\%) had used some type of fitness application. The most frequently downloaded applications were exercise plans, such as Weider's Aerobic Six and applications for physical activity monitoring (steps, distance), such as Endomondo. Exergames were less popular. Only 5.29\% of the respondents claimed they used exergames regularly, although about every third person used them regularly, especially males. Conclusions. Eight of ten respondents held the view that such applications are useful but are something that they could do without; only one in ten could not imagine exercising without such applications. Most of the latter category of users were persons at the action stage, next - persons at the preparation stage, but - what is interesting - none at the maintenance stage. It seems, then, that such applications may be - and in the light of the data obtained really are - an indispensable aid for people at the early stages of developing a habit of regular physical activity.
\end{abstract}

Key words: youth, mobile applications, exergaming, physical activity

\section{Introduction}

Experts unanimously agree that the lack of physical activity is a serious public health problem, playing an important role in the etiology of many diseases, such as hypertension, type 2 diabetes mellitus, and coronary heart disease, to name but a few [1]. Active behaviours in different contexts (recreation and leisure, locomotion, etc.) are promoted by means of diverse tools and strategies. One way of supporting physical activity and other health behaviours can be the use of mobile applications keeping track of physical activity, providing information on how to perform exercise, or helping motivate the exerciser by such selfregulatory strategies as goal setting $[2,3,4]$. Even considering that the reliability of at least some of their functions is questionable (see, for example, the study by Bergman et al. 2012 [5]), at the same time we should not underestimate their potential role in triggering and strengthening physical activity behaviours.

A similar role in promoting active behaviours may be fulfilled by another invention of computer technologies - games that are controlled by a player's body movements. Computer games, along with television, are frequently blamed for the decreasing physical activity of children and youth [6] and according to a WHO Collaborative Study [7], in 2010, nearly half of young people spent over 2 hours playing computer games on weekdays, and on weekends this proportion increased to $67 \%$. Such features of computer/video games as high availability, the immediacy of gratification in the form of emotional arousal, and their popularity with peers, among others, make them a more attractive alternative to physical activity, the behavioural cost of which is usually much higher, demanding 'higher activation energy', as Csikszentmihayi defines it [8]. Exergames (a blending of 'exercise' and 'games') are an attempt to reconcile physical activity and computer entertainment. These games are designed in a manner that demands the player make intense, vigorous body movements to trigger the desired effect on the screen. Since the end of the 1990s, when the first such game appeared on the market (Dance Dance Revolution from Konami), exergames have become more and more popular and are even considered as a teaching tool in physical education [9], or at least one of the modern means to compensate for decreasing physical activity in children and youth. McDougall and Duncan [10] found that children who play PlayStation active games even for a short time but do so daily can achieve the recommended level of physical activity.

As far as we know, the phenomenon of using fitness mobile applications and exergames by Polish youth has not been studied. Few studies have explored the physiological or motor aspects of exergames [11, 12]. Thus, the aim of the present study was to investigate to what extent the abovementioned technologies are used by high-school youth and how this use relates to their physical activity. The latter was conceptualised as stages of motivational readiness for exercise or stages of change as pro- 
posed by the transtheoretical model [13]. Stages of change are intentional or behavioural states that describe 'distance' to the desirable end point in the form of regular, habitual physical activity. Usually five such stages are mentioned: precontemplation (a person is inactive and is not planning to start being active in the immediate future), contemplation (a person is inactive but is thinking about becoming active), preparation (a person is active but irregularly, below the recommended level), action (a person is regularly active but has not yet maintained this behaviour for at least 6 months, so the behaviour is not yet a habit), maintenance (a person is regularly active and has maintained that activity for the past 6 months or more, so it can be said that it has become a kind of relatively established habit).

\section{Material and methods}

A total of 435 adolescents (including 263 girls), aged 17.78 \pm 1.19 years agreed to participate in the survey. Participants were asked to fill in an anonymous questionnaire consisting of 12 questions assessing whether or not and how often they used mobile applications and exergames and of what type as well as an ordered categorical scale measuring the stages of (exercise) change developed by Marcus, Rakowski, and Rossi [14]. The latter consisted of five items which were as follows: "I currently do not exercise, and I do not intend to start in the next six months" (precontemplation), "I currently do not exercise, but I am thinking about starting to exercise within the next six months" (contemplation), "I currently do some exercise but not regularly" (preparation), "I currently exercise regularly but have only begun doing so within the past six months" (action), and "I currently exercise regularly and have done so for longer than six months" (maintenance).

Descriptive statistics (means and standard deviations) were used to describe the data obtained and to test differences between groups; a chi ${ }^{2}$ test was used with Cramér's V statistic as a measure of effect size in contingency tables larger than $2 \times 2$. It was assumed that a V-value under 0.30 means small effect size (weak association between variables), a value between 0.30 and 0.50 indicates medium effect size (moderate association), and a value above 0.50 means large effect size (strong association) [15].

\section{Results}

\section{Stages of exercise change}

The distribution of individuals across the stages of change indicated that nearly half of the participants, especially girls, were irregularly active (preparation), and approximately $1 / 3$ were active in a regular manner (action and maintenance). The proportion of participants in each stage of change was related to sex with a rather weak correlation. The biggest proportional differences between girls and boys in particular stages concerned the contemplation stage, for which there were nearly twice as many boys as girls, and the maintenance stage, with quite the opposite proportions. The exact data on the distribution of participants in terms of the stages of change are displayed in table 1.

\section{Use of mobile fitness applications}

The highest proportion of participants, $82.53 \%(n=359)$, had mobile phones with the Android operating system, then with WindowsPhone $(\mathrm{n}=34 ; 7.82 \%)$, and then with IOS ( $\mathrm{n}$ $=24 ; 5.06 \%$ ). The remaining $4.59 \%$ had mobile devices with other systems. Accordingly, most mobile applications were downloaded from the Google Play store $(n=362 ; 83.22 \%)$, the Windows Phone Store $(\mathrm{n}=31 ; 7.13 \%)$, and the App Store $(\mathrm{n}=$ $30 ; 6.89 \%)$. Few participants indicated other sources, such as the Nokia store, the file sharing website chomikuj.pl, and other websites.

There were 134 participants who used applications from the fitness category, of whom 53 persons (12.18\%) were current users and 81 persons were ex-users $(18.62 \%)$. The use of fitness applications was not related to sex: the difference between the proportion of female users ( $\mathrm{n}=73 ; 27.76 \%$ of the girls) and male users ( $n=61 ; 35.46 \%$ of the boys) was insignificant, with a negligible effect size: $\mathrm{chi}^{2}{ }_{(\mathrm{df}=2)}=3.06 ; \mathrm{p}=0.216$; Cramér's $\mathrm{V}=0.083$.

As expected, the proportion of fitness applications users was highest among regular exercisers, reaching the levels of $43.95 \%$ in the maintenance stage and $48.33 \%$ in the action stage. Interestingly, persons who had just started performing regular physical activity (action stage) were more frequently current application users than maintainers. These data may imply that those who start their regular activity have a stronger desire to use applications than persons whose physical activity behaviours are habitual. It is also worth noting that among users (or at least

Table 1. Distribution of participants according to the stages of change

\begin{tabular}{|c|c|c|c|c|c|c|c|}
\hline \multirow{2}{*}{ Stage of change } & \multicolumn{2}{|c|}{ Total } & \multicolumn{2}{|c|}{ Boys } & \multicolumn{2}{|c|}{ Girls } & \multirow{2}{*}{ Comparison between boys and girls } \\
\hline & n & $\%$ & n & $\%$ & $\mathrm{n}$ & $\%$ & \\
\hline Pc & 36 & 8.28 & 19 & 7.2 & 17 & 9.88 & \multirow{5}{*}{$\begin{array}{c}\mathrm{chi}^{2}{ }_{(\mathrm{df}=4)}=18.33 ; p=0.001 ; \\
\text { Cramér's V = } 0.21\end{array}$} \\
\hline C & 54 & 12.41 & 41 & 15.59 & 13 & 7.56 & \\
\hline $\mathrm{Pp}$ & 194 & 44.60 & 126 & 47.91 & 68 & 39.53 & \\
\hline Ac & 60 & 13.79 & 37 & 14.07 & 23 & 13.37 & \\
\hline Mt & 91 & 20.92 & 40 & 15.21 & 51 & 29.65 & \\
\hline
\end{tabular}

Note: $\mathrm{Pc}=$ precontemplation $; \mathrm{C}=$ contemplation $\mathrm{Pp}=$ preparation $; \mathrm{Ac}=$ action; $\mathrm{Mt}=$ maintenance . 


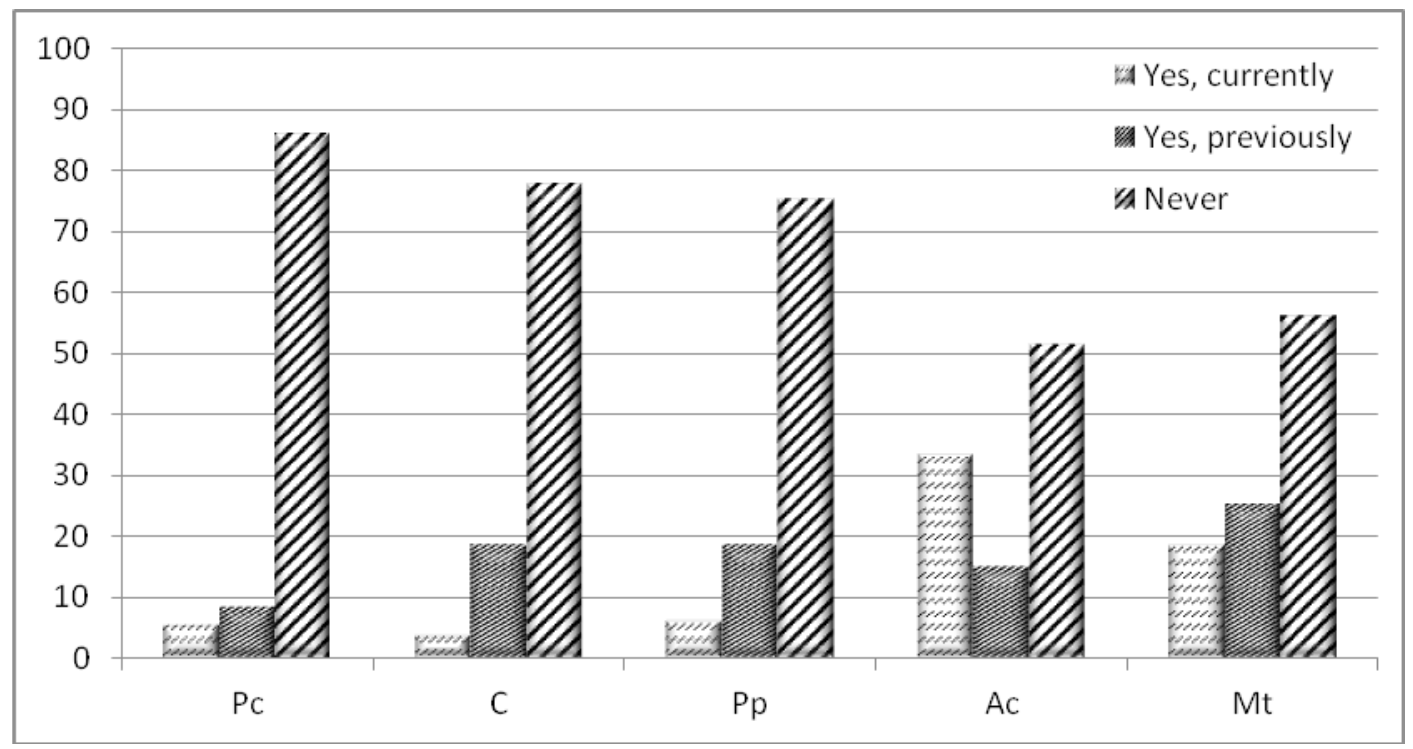

Figure 1. Use of fitness mobile applications and the stages of change

holders) of fitness applications, there were a few (exactly five) inactive adolescents. Only two respondents from this group specified which applications they used, and in both these cases, these were exercise sets for abdominals. Detailed data on mobile application use by stage of change are illustrated in figure 1. The differences between the stages were significant with small effect size: $\mathrm{chi}^{2}{ }_{(\mathrm{df}=8)}=48.06 ; \mathrm{p}<0.001$; Cramér's $\mathrm{V}=0.235$.

The most frequently used fitness applications belonged to two categories: exercise plans/exercise sets (especially Weider's Aerobic Six, $n=15$, and ABS, $n=15$ ) that were indicated by 73 (16.77\%) persons (40 girls and 33 boys) and movement trackers (especially Endomondo, n = 48) chosen by $72(16.55 \%)$ persons (42 girls and 32 boys). A lower proportion of participants declared their use of applications belonging to the category of exercise motivators ( $\mathrm{n}=9 ; 2.07 \%$, including 3 girls $)$ and personalised diet plans ( $\mathrm{n}=8 ; 1.84 \%$, including 3 girls). Twenty-one adolescents can be described as multi-app users, downloading and using at least two fitness applications simultaneously. The most popular ones were free applications, chosen by $74.63 \%$ of users, while only $25.37 \%$ used paid applications or paid versions of applications that were also available free of charge.

Interestingly, only one-tenth $(\mathrm{n}=14 ; 10.45 \% ; 5$ boys and 9 girls) of fitness application users perceived them as essential support for their physical activity; for the vast majority $(\mathrm{n}=106$; $79.10 \%$; 49 boys, 57 girls), they were useful but not necessary, whereas for $10.45 \%$, (7 girls, 7 boys) they were absolutely inessential, and these persons could do without them. No significant differences were found between the sexes in the perceived usefulness of the applications: $\mathrm{chi}^{2}{ }_{(\mathrm{df}=2)}=0.677 ; \mathrm{p}=0.713$.

Nearly 3/4 (74.24\%) of fitness applications users had a definitive view concerning their reliability. They were considered to be a very reliable tool by only $14.39 \%$ of participants, especially current users (26.42\% compared to $6.17 \%$ of ex-users). For $10.61 \%$ of users, these applications were not particularly reliable (including $13.21 \%$ of current users and $12.35 \%$ of past users).

\section{Use of exergames by adolescents}

Regular use of exergames was declared by only 23 participants (5.29\%), including 14 girls (5.32\%) and 9 boys (5.23\%), and occasional use was mentioned by 150 respondents $(34.48 \%)$, including 78 girls (29.66\%) and 72 (41.86\%) boys. The difference between the distribution of regular users, occasional users, and nonusers of exergames among boys and girls was significant, however, with weak size effect: $\mathrm{chi}^{2}{ }_{(\mathrm{df}=2)}=7.40 ; \mathrm{p}=0.025$, Cramér's $\mathrm{V}=0.131$. The most frequently mentioned exergames were Virtual Tennis, Kinect Sport, RunAway Sport, Move Sport (champions sport), Fitness, Beach Sports, Dance Revolution, Wii Sports, Just Dance, and Star Wars III.

Every fourth respondent $(\mathrm{n}=114 ; 26.21 \%)$ agreed that exergames may replace more traditional forms of physical activity, $38.16 \%$ thought that this was impossible, and the rest held no view on this matter. The respondents' perception of interchangeability between exergames and traditional forms of physical activity was not related to sex: $\mathrm{chi}^{2}{ }_{(\mathrm{df}=2)}=3.83 ; \mathrm{p}=0.147$.

Nearly every second respondent $(n=80 ; 46.24 \%)$ had at least one exergame of their own, while the rest played them in their peers' houses $(n=99)$, and to a lesser extent in electronics stores, in their close or distant family, in their school, and in fitness clubs.

\section{Conclusions}

The main purpose of the study was to assess the extent to which high-school students use modern technology aimed at the recording and promotion of physical activity, that is mobile applications that help to track, plan, and perform exercise and computer/video games that require (sometimes intense) movement to trigger a desired action in the screen. The findings of the study showed that regardless of sex, nearly every third respondent currently used mobile applications from the Health \& Fitness category or had used them at some point in the past. Proportionally, however, there were many more ex-users than current users. As expected, the highest proportion of users was found among respondents that were regularly active (at the action and maintenance stages of motivational readiness to physical activity), among whom the said applications were used by nearly every second person.

The most frequently used applications were workout plans/ exercise sets (such as Weider's Aerobic Six) and tools for record- 
ing movement (steps, distance) and related parameters, such as calories burned. In the latter category, the prevailing application was Endomondo, which allows not only recording different aspects of the user's physical activity, but also sharing personal data with other users via social media such as Facebook or Twitter.

Eight of ten respondents held the view that such applications were useful, but were something that they could do without, and only for one in ten were such applications something they could not imagine exercising without. Most of the latter category of users were persons in the action stage, next - persons in the preparation stage, but, interestingly, none from the maintenance stage. It seems, then, that such applications may be - and in the light of the data obtained really are - an indispensable aid for people at the early stages of developing a habit of regular physical activity.

The second tool with the potential to motivate people to be more physically active is the so-called exergame, which was perceived not only as a more favourable alternative - because of the fact that it is active - to more traditional (sedentary) computer games, but also as a means of compensating for movement deficits in youth $[8,11,16,17]$. However, exergames were not very popular with - or available to - the respondents. Only a small number of subjects played them regularly, although about onethird played them occasionally. Playing exergames was related to sex, with boys being more frequent users.

To our knowledge, this is the first study in Poland to investigate the phenomenon of using mobile applications to enhance physical activity and exergames in high-school pupils. Moreover, our results fill the gap in the knowledge on the use of the latest technological developments in managing physical activity behaviours among high-school pupils.

This study has several limitations that should be mentioned. First, the study protocol does not allow us to establish any cause-effect relations between variables. Second, the study depicts only a part of reality which is not necessarily representative for the whole population. Finally, the use of self-reported data is also prone to bias with regard to socially desirable responses.

\section{Literature}

1. Dishman R., Washburn R., Heath G. (2004). Physical activity epidemiology. Champaign: Human Kinetics Publishers.

2. Middelweerd A., Mollee J., van der Wal N., Brug J., Te Velde S. (2014). Apps to promote physical activity among adults: A review and content analysis. International Journal of Behavioral Nutrition and Physical Activity 11, 97. DOI: 10.1186/ s12966-014-0097-9.
3. Conroy D., Yang Ch., Maher J. (2014). Behavior change techniques in top-ranked mobile apps for physical activity. American Journal of Preventive Medicine 46(6), 649-652.

4. Vickey T., Breslin J., Williams A. (2013). Fitness - There's an app for that: Review of mobile fitness apps. The International Journal of Sport and Society 3, 109-127.

5. Bergman R., Spellman J., Hall M., Bergman S. (2012). Is there a valid app for that? Validity of a free pedometer iPhone application. Journal of Physical Activity and Health 9, 670676.

6. Janicki G. (2009). New aspects of leisure at the turn of the century. In Z. Dziubiński, K. Jankowski (eds), Physical culture in modern society (pp. 380-391). Warszawa: SALOS. [in Polish]

7. Mazur J., Małkowska-Szkutnik A. (ed.) (2011). The results of the HSBC 2010 study. Technical report. Warszawa: Instytut Matki i Dziecka. [in Polish]

8. Csikszentmihalyi M. (1998). Finding flow in everyday life. Warszawa: W.A.B. [in Polish]

9. Meckbach J., Gibbs B., Almqvist J., Öhman M., Quennerstedt M. (2013). Exergames as a teaching tool in physical education? Sport Science Review 22(5-6), 369-385. DOI: 10.2478/Ssr-2013-0018.

10. McDougall J., Duncan M. (2008). Children, video games and physical activity: An exploratory study. International Journal on Disability and Human Development 7(1), 89-94.

11. Polechoński J., Tomik R., Dobias M. (2014). Use of a motion-controlled video game to evaluate the reaction time in children aged 11-13. Rozprawy Naukowe Akademii Wychowania Fizycznego we Wroctawiu 44, 93-98. [in Polish]

12. Polechoński J., Groffik D., Zając-Gawlak I., Machwic A. (2010). Physical activity during a dance computer game. In D. Umiastowska (ed.), Physical activity of people in different ages (pp. 171-181). Szczecin: USz and PTN KF. [in Polish]

13. Prochaska J., Velicer W. (1997). The transtheoretical model of health behavior change. American Journal of Health Promotion 12(1), 38-48.

14. Marcus B.H, Forsyth L. (2009). Motivating people to be physically active. Champaign: Human Kinetics Publishers.

15. Speed H.D., Andersen M.B. (2000). What exercise and sport scientists don't understand. Journal of Science and Medicine in Sport 3(1), 84-92.

16. Perron R., Graham C., Feldman J., Moffett R., Hall E. (2011). Do exergames allow children to achieve physical activity intensity commensurate with national guidelines? International Journal of Exercise Science 4(4), 257-264.

17. Meckbach J., Gibbs B., Almqvist J., Quennerstedt M. (2014). Wii Teach movement qualities in physical education. Sport Science Review 23(5-6), 241-266. DOI: 10.1515/ssr-20150004.

Submitted: August 21, 2015

Accepted: August 30, 2016 